\title{
A crucial role for thiol antioxidants in estrogen-deficiency bone loss
}

\author{
Jenny M. Lean, Julie T. Davies, Karen Fuller, Christopher J. Jagger, Barrie Kirstein, \\ Geoffrey A. Partington, Zoë L. Urry, and Timothy J. Chambers \\ Department of Cellular Pathology, St. George's Hospital Medical School, London, United Kingdom
}

\begin{abstract}
The mechanisms through which estrogen prevents bone loss are uncertain. Elsewhere, estrogen exerts beneficial actions by suppression of reactive oxygen species (ROS). ROS stimulate osteoclasts, the cells that resorb bone. Thus, estrogen might prevent bone loss by enhancing oxidant defenses in bone. We found that glutathione and thioredoxin, the major thiol antioxidants, and glutathione and thioredoxin reductases, the enzymes responsible for maintaining them in a reduced state, fell substantially in rodent bone marrow after ovariectomy and were rapidly normalized by exogenous $17-\beta$ estradiol. Moreover, administration of $N$-acetyl cysteine (NAC) or ascorbate, antioxidants that increase tissue glutathione levels, abolished ovariectomy-induced bone loss, while L-buthionine-(S,R)-sulphoximine (BSO), a specific inhibitor of glutathione synthesis, caused substantial bone loss. The 17- $\beta$ estradiol increased glutathione and glutathione and thioredoxin reductases in osteoclast-like cells in vitro. Furthermore, in vitro NAC prevented osteoclast formation and NF- $\mathrm{KB}$ activation. BSO and hydrogen peroxide did the opposite. Expression of TNF- $\alpha$, a target for NF- $\kappa B$ and a cytokine strongly implicated in estrogen-deficiency bone loss, was suppressed in osteoclasts by $17-\beta$ estradiol and NAC. These observations strongly suggest that estrogen deficiency causes bone loss by lowering thiol antioxidants in osteoclasts. This directly sensitizes osteoclasts to osteoclastogenic signals and entrains ROS-enhanced expression of cytokines that promote osteoclastic bone resorption.
\end{abstract}

J. Clin. Invest. 112:915-923 (2003). doi:10.1172/JCI200318859.

\section{Introduction}

Postmenopausal osteoporosis is a disease of high prevalence and a cause of substantial morbidity and mortality. It is due to estrogen deficiency, which leads to bone loss through increased osteoclastic function (1). The mechanisms through which estrogen deficiency stimulates bone resorption remain controversial. Direct effects on osteoclasts (2-4), upregulation of the osteoclast-inductive cytokine RANK ligand (RANKL) (5), or downregulation of its decoy receptor osteoprotegerin in osteoblasts (6) have been reported. In addition, several inflammatory cytokines have been implicated: estrogen has been reported to suppress expression of TNF- $\alpha$, IL-1, and IL- 6 in osteoclast-supportive bone marrow stromal cells, monocytes, and lymphocytes (see refs. 1, 7, 8 for reviews).

In other tissues, the beneficial effects of estrogen, such as those on lipids, endothelial cells, and neurons, are

Received for publication May 7, 2003, and accepted in revised form July 8, 2003

Address correspondence to: T.J. Chambers, Department of Cellular Pathology, St. George's Hospital Medical School, Cranmer Terrace, London SW17 0RE, United Kingdom.

Phone: 44-20-8725-5270; Fax: 44-20-8725-0064;

E-mail: t.chambers@sghms.ac.uk.

Conflict of interest: The authors have declared that no conflict of interest exists.

Nonstandard abbreviations used: RANK ligand (RANKL); reactive oxygen species (ROS); c-Jun amino-terminal kinase (JNK); L-buthionine-(S,R)-sulphoximine (BSO); $N$-acetyl cysteine (NAC); tartrate-resistant acid phosphatase (TRAP);

electrophoretic mobility shift assay (EMSA). considered to occur through improved defense against oxidative stress (9-12). We hypothesized that estrogen might similarly augment oxidant defenses in bone. Such an augmentation of oxidant defenses would be expected to lower the concentration of reactive oxygen species (ROS) within cells. Although at high concentrations ROS damage many cell constituents, they also affect many signaling proteins at levels considerably lower than those that cause oxidative injury $(13,14)$. Signaling cascades can be modulated by ROS following oxidant stress- or cytokine-regulated generation of ROS or through modulation of oxidant defenses $(13,15,16)$. Therefore, if estrogen does increase oxidant defenses in bone, this might modulate signal transduction in bone cells with signaling pathways that are sensitive to ROS.

One such cell is the osteoclast, which has been shown to be activated by ROS $(17,18)$. Several signals essential for osteoclasts are sensitive to activation by ROS, including NF- $\mathrm{\kappa B}$, c-Jun amino-terminal kinase (JNK), PI3K, and p38 MAP kinase (see refs. 13, 15, 19). Moreover, osteoclasts contain a NADPH oxidase (20), an enzyme that is capable of cytokine-regulated generation of ROS. ROS are also potent inducers in many cells of TNF- $\alpha$, and other cytokines strongly implicated in the bone loss of estrogen deficiency. Thus, if estrogen increases oxidant defenses in bone, estrogen deficiency might directly or indirectly stimulate osteoclastic bone resorption.

The effects of estrogen on antioxidant defenses in bone have not been investigated. The major determinants of the reductive state of soluble proteins are glu- 
tathione and thioredoxin (see refs. 16, 21, 22). Upon oxidation, their oxidized forms are reverted to the reduced state by glutathione reductase and thioredoxin reductase, respectively. We found substantial decreases in glutathione and thioredoxin and in the enzymes that regenerate their reduced forms in rodent bone marrow. These decreases were reversed by estrogen administration. We also found that administration of antioxidants completely prevented bone loss in ovariectomized mice, while L-buthionine-(S,R)-sulphoximine (BSO), a specific inhibitor of glutathione synthesis, depleted glutathione and caused substantial osteolysis in ovaryintact mice. In vitro, estrogen augmented osteoclastic expression of glutathione and thioredoxin reductases. Furthermore, as evidence of a mechanistic relationship between ROS and the hyper-resorption of estrogen deficiency, we found that BSO enhanced and $N$-acetyl cysteine (NAC), an antioxidant and a glutathione precursor (16), suppressed NF-кB activity, while estrogen and NAC suppressed TNF- $\alpha$ expression in osteoclasts.

\section{Methods}

Media and reagents. Bone marrow cells were incubated in MEM with Earle's salts (Sigma-Aldrich, Poole, Dorset, United Kingdom) supplemented with 10\% FBS (Autogen Bioclear UK Ltd., Calne, United Kingdom) and 2 $\mathrm{mM}$ glutamine, $100 \mathrm{IU} / \mathrm{ml}$ benzylpenicillin, and 100 $\mu \mathrm{g} / \mathrm{ml}$ streptomycin (Sigma-Aldrich). Incubations were performed at $37^{\circ} \mathrm{C}$ in $5 \%$ carbon dioxide in humidified air. Recombinant human (rh)M-CSF was provided by Chiron Corp. (Emeryville, California, USA); soluble rhRANKL was from Insight Biotechnology (Wembley, Middlesex, United Kingdom). All other reagents were obtained from Sigma-Aldrich unless otherwise stated.

Animals. The animals used were female 6- to 8-week-old Wistar rats or MF-1 mice from the St. George's Hospital Medical School colony unless otherwise stated.

Assessment of effect of ovariectomy and estrogen on oxidant defenses. Groups of six rats or mice were subjected to ovariectomy or a sham operation, followed by pair feeding. Three weeks later, a single dose of $17-\beta$ estradiol $(10 \mu \mathrm{g} / \mathrm{kg})$, or $17-\alpha$ estradiol $(100 \mu \mathrm{g} / \mathrm{kg})$, or vehicle was administered subcutaneously in corn oil. All animals were killed 24 hours later. Success of the ovariectomy was confirmed by absence of ovaries and atrophy of uteri. Femora were rapidly cleaned and bone marrow harvested into ice-cold heparinized water and homogenized using a Polytron homogenizer. Liver and spleen were weighed and homogenized in ice-cold water. The homogenates were divided into two equal parts. To one, 0.1 vol of $1 \%$ Triton $\mathrm{X}-100$ was added; to the other, an equal volume of $10 \%$ sulfosalicylic acid was added. The Triton extract was centrifuged at $10,000 \mathrm{~g}$ for 10 minutes at $4{ }^{\circ} \mathrm{C}$, and the supernatant was used for enzyme assays. Protein concentration was determined using Coomassie blue (Pierce, Tattenhall, United Kingdom), with BSA as protein standard.

Glutathione was measured in the samples after deproteinization with sulfosalicylic acid. Total glu- tathione (reduced glutathione [GSH] + oxidized glutathione [GSSG]) was measured using the GSH reductase-dithionitrobenzoic acid (DTNB) recycling procedure according to Tietze (23). GSSG was assayed as above after derivatization of GSH in the sample with 2-vinylpyridine (24). Glutathione reductase was assayed with a kit from Calbiochem (La Jolla, California, USA) according to the manufacturer's instructions. Thioredoxin and thioredoxin reductase were assayed by the NADPH-dependent reduction of DTNB at $412 \mathrm{~nm}$ in the insulin-reducing assay (25).

Effect of NAC and ascorbate on ovariectomy-induced bone loss. Mice (six per group) were subjected to ovariectomy or sham ovariectomy. NAC $(100 \mathrm{mg} / \mathrm{kg} /$ day intraperitoneally) was administered twice per day. Ascorbate was administered at a dose sufficient to increase tissue glutathione levels (26). For this, $1 \mathrm{mmol} / \mathrm{kg}$ or vehicle were administered intraperitoneally at 7:00 am and 6:00 pm each day. Ascorbate was dissolved immediately before use in isosmolar ice-cold saline and adjusted to $\mathrm{pH} 6.8$ with $2 \mathrm{M} \mathrm{NaOH}$. After 14 days, animals were killed, and bone was prepared for assessment of bone volume, analysis of parameters of bone resorption, and bone formation, as described (27). There was no significant change in the weight of mice during the experimental period. Ovariectomy was confirmed by uterine atrophy. Effect of BSO on mouse bone. Groups of six female mice (Harlan Olac Ltd., Oxon, United Kingdom) were administered BSO (2 mmol/ $\mathrm{kg}$ intraperitoneally) at 7:00 am and 6:00 pm each day for 3 weeks. BSO was also included in the drinking water $(20 \mathrm{mM})$. Calcein was injected 1 and 6 days before killing the animals. Bones were processed for static and dynamic analysis as described (27).

Effect of $17-\beta$ estradiol on osteoblastic cells. Calvarial osteoblasts were obtained from neonatal female rats. Calvariae were dissected free of periosteum and associated soft tissues and incubated in MEM containing bacterial collagenase $\left(1 \mathrm{mg} / \mathrm{ml}\right.$, type II) at $37^{\circ} \mathrm{C}$ for 100 minutes. The tissue fragments were then agitated, and suspended cells were removed and centrifuged at $250 \mathrm{~g}$ for 3 minutes. The cells were resuspended in MEM/FBS $\left(2 \times 10^{5}\right.$ cells $\left./ \mathrm{ml}\right)$ in $75-\mathrm{cm}^{2}$ flasks and incubated until confluent. Cells were then resuspended in trypsin/EDTA, washed, and incubated at $4 \times 10^{5}$ cells/well in six-well plates (Helena Biosciences Europe, Sunderland, Tyne and Weir, United Kingdom) in MEM/FBS for 24 hours. Medium was then removed and incubation was continued for 18 hours in 17- $\beta$ estradiol $\left(10^{-9} \mathrm{M}\right)$ or vehicle, in phenol red-free MEM, and 10\% charcoal-stripped FBS. To prepare charcoal-stripped serum, FBS was incubated with activated charcoal $(10 \mathrm{mg} / \mathrm{ml})$ for 2 hours at $37^{\circ} \mathrm{C}$. The serum was then centrifuged and the supernatant passed through a $0.22-\mu \mathrm{m}$ filter (Millipore, Molsheim, France).

Osteoblast-like UMR 106 cells and MC3T3-E1 cells were added to six-well plates $\left(4 \times 10^{6}\right.$ cells/well $)$ and similarly allowed to settle for 24 hours in MEM/FBS before 
replacement of medium with vehicle/17- $\beta$ estradiol, phenol red-free MEM, and charcoal-stripped serum.

Cells were then scraped into suspension with a cell scraper and assayed for total glutathione and glutathione reductase, as described above.

Effects on in vitro-generated osteoclasts. Osteoclasts were generated from nonadherent murine bone marrow cells as described previously (28). Briefly, bone marrow cells were obtained from 3- to 4-week-old female MF-1 mice and incubated for 24 hours in M-CSF $(5 \mathrm{ng} / \mathrm{ml})$ at $3 \times 10^{5}$ cells $/ \mathrm{ml}$. This incubation of bone marrow cells at low density in M-CSF for 24 hours efficiently depletes the cell preparations of stroma: stromal cells were not seen at any stage in the cultures, and no cells survived in cultures subsequently incubated without M-CSF. After 24 hours, nonadherent bone marrow cells were washed, resuspended, and incubated in MEM/FBS with M-CSF (50 ng/ml) and RANKL (30 $\mathrm{ng} / \mathrm{ml}$ ) for 5 days. Cultures were fed every 2-3 days.

To test the effect of estrogen on thiol antioxidant mechanisms, osteoclasts were generated in six-well plates for 5 days. Medium was then removed, and incubation was continued for 18 hours in M-CSF, RANKL, and $17-\beta$ estradiol $\left(10^{-9} \mathrm{M}\right)$, ICI 182,780 $\left(10^{7} \mathrm{M}\right.$; Tocris Cookson Ltd., Bristol, United Kingdom), or vehicle, in phenol red-free MEM and 10\% charcoal-stripped FBS. Cells were scraped into suspension with a cell scraper and assayed for oxidized and reduced glutathione and glutathione, and thioredoxin reductases, as described above.

For assessment of the effect of agents on osteoclastic differentiation, nonadherent bone marrow cells were incubated for 5 days in the wells of 96 -well plates (Helena Biosciences) in RANKL, M-CSF, with or without BSO $(10 \mu \mathrm{M})$, NAC $(30 \mathrm{mM})$, or hydrogen peroxide (1 $\mu \mathrm{M})$. Cells were then fixed in $10 \%$ formalin for $10 \mathrm{~min}$ utes and stained for tartrate-resistant acid phosphatase (TRAP). For this, cells were permeabilized in acetone for 10 minutes, washed, and stained for acid phosphatase in the presence of $0.05 \mathrm{M}$ sodium tartrate. The substrate used was napthol AS-B1 phosphate. The number of TRAP-positive cells containing at least three nuclei was counted microscopically using an eyepiece graticule.

For assessment of NF-кB activation, osteoclast cultures were washed and incubated in M-CSF plus BSO $(100 \mu \mathrm{M})$ or NAC $(30 \mathrm{mM})$ for 2 hours, before readdition of RANKL. Cells were harvested by scraping 30 minutes later. Nuclear extracts were prepared as described previously (29), except that the cysteine protease inhibitor trans-epoxysuccinyl-1-leucylamido(4guanidino)-butane (Sigma-Aldrich), was included in the LS buffer (20 nM HEPES pH 7.9, $2 \mathrm{mM} \mathrm{MgCl}_{2}$ ) at a final concentration of $3 \mathrm{nM}$, and, in addition, microcystin-LR (Qbiogene-Alexis, Bingham, Nottinghamshire, United Kingdom) was included at a final concentration of $4 \mathrm{nM}$. The cells were washed in situ twice with cold PBS, once with cold LS buffer, and lysed by the addition of LS buffer containing $0.1 \%$ Triton $\mathrm{X}-100$ with gentle scraping with a cell scraper.
The protein concentration in nuclear extracts was determined using the bicinchoninic acid protein assay reagent (Pierce.), and an electrophoretic mobility shift assay (EMSA) was carried out using aliquots containing equal amounts of protein ( $4 \mu \mathrm{g}$ per assay). The

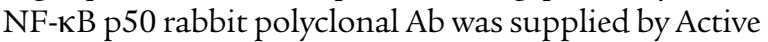
Motif LLC (Rixensart, Belgium). The following modifications were made to the previously published EMSA procedure (29). For the Supershift assay the nuclear extracts were incubated for 30 minutes at $4^{\circ}$ with the $\mathrm{Ab}(2 \mu \mathrm{l}$ per assay) prior to the addition of the probe and poly(dIdC). The protein-DNA complexes were resolved on a $4 \%$ polyacrylamide gel in $0.5 \times$ tris-glycineEDTA (TGE) buffer for 2 hours at $4^{\circ} \mathrm{C}$ at $12.5 \mathrm{~V} / \mathrm{cm}$ following the protocol recommended by Active Motif LLC. The NF-кB oligonucleotide probe 5'-AGTTGAGGGGACTTTCCCAGG was supplied by Promega UK Ltd. (Southampton, United Kingdom). The mutant NF-кB oligonucleotide 5'-GCCATGGGCCGATCCCCGAAGTCC was supplied by Active Motif.

For assessment of the effect of $17-\beta$ estradiol on osteoclastic TNF- $\alpha$ mRNA expression, osteoclasts were generated as above in $75-\mathrm{cm}^{2}$ flasks. After 5 days, the medium was discarded and replaced with phenol red-free MEM containing 10\% charcoal-stripped serum and $\mathrm{M}-\operatorname{CSF}(50 \mathrm{ng} / \mathrm{ml})$, together with $17-\beta$ estradiol $\left(10^{-9} \mathrm{M}\right)$ or vehicle. After incubation for 18 hours, RANKL ( 30 $\mathrm{ng} / \mathrm{ml}$ ) was added. Cells were incubated for a further 3 hours and then harvested for analysis of RNA. For this, total RNA was extracted from cultures on plastic using TRIzol (Invitrogen Ltd., Paisley, United Kingdom). Total RNA $(25-40 \mu \mathrm{g})$ was size separated in a $1.2 \%$ agarose gel and blotted as described previously (30). Probes were labeled by Megaprime DNA-labeling system (Amersham International, Amersham, United Kingdom) with $\alpha-32$ P (ATP) (Amersham International). Blots were hybridized with probes for murine TNF- $\alpha$ and $\beta$-actin. The murine TNF- $\alpha$ probe was obtained by PCR using sense primer 5'-CCCCAAAGGGATGAGAAGTT and antisense primer 5'-CTTAGACTTTGCGGAGTCCG (Genbank accession no. XM_110221); murine $\beta$-actin was obtained using the sense primer 5'-GTCATCACTATTGGCAACGA and antisense primer 5'-CCTGTCAGCAATGCCTGGGT (Genbank accession no. M12481)

For assessment of the effect of the effect of NAC on osteoclastic TNF- $\alpha$ expression, osteoclasts were generated as above in $75-\mathrm{cm}^{2}$ flasks. After 5 days, the medium was discarded and replaced with fresh medium containing M-CSF $(50 \mathrm{ng} / \mathrm{ml})$ and RANKL (30 $\mathrm{ng} / \mathrm{ml})$ with or without NAC $(30 \mathrm{mM})$. The cells were harvested after 3 more hours for analysis of TNF- $\alpha$ mRNA expression as above.

Statistical analysis was by ANOVA (Fisher's protected least-squares difference [PLSD] test) for multiple comparisons and the Student $t$ test for paired comparisons.

\section{Results}

The total and reduced glutathione content and the concentration of thioredoxin in rat bone marrow 

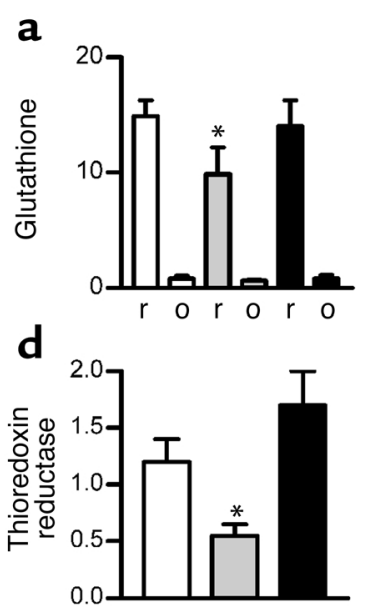
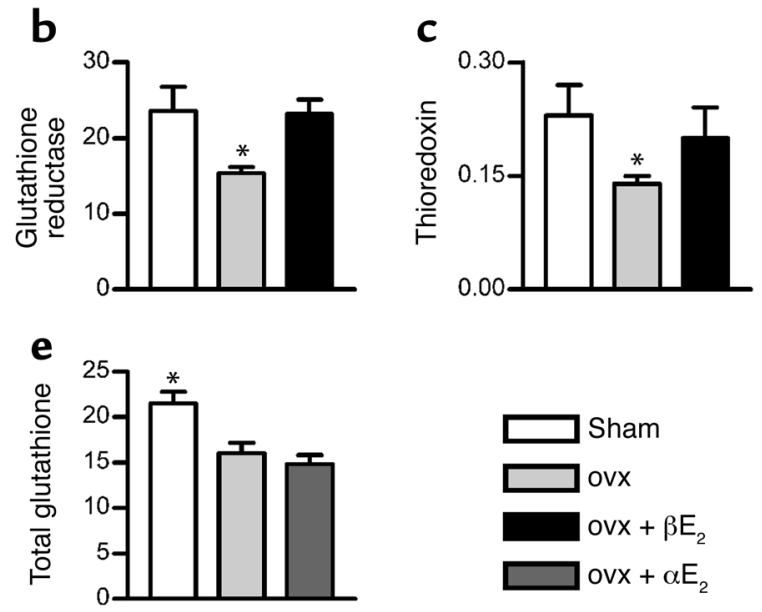

antioxidants normalized the ovariectomy-induced increase in the numbers of osteoclasts on bone surfaces, the extent of bone surface covered by osteoclasts, and the extent of eroded surface. Osteoblast indices were also restored to normal. As a further test of the notion that thiol antioxidants can regulate bone resorption in vivo, we tested the effect BSO, a specific inhibitor of the enzyme that is rate limiting for glutathione synthesis, on mouse bone. Total glutathione fell significantly in the bone marrow of BSO-treated mice, to an extent comparable to that observed after ovariectomy (from $14.5 \pm$ 1.1 to $6.8 \pm 0.1 \mathrm{nmol} / \mathrm{mg}$ protein). In contrast to the effects of the antioxidants, BSO administration caused substantial bone loss (Figure 3). This was associated with an increase in the number of

were all significantly decreased by ovariectomy (Figure 1). Glutathione reductase, the enzyme responsible for regenerating glutathione from oxidized glutathione, and thioredoxin reductase, the enzyme responsible for regenerating thioredoxin, were also substantially lower in bone marrow from ovariectomized rats. The levels of all of these components of the thiol antioxidant system were rapidly normalized by a single dose of $17-\beta$ estradiol $(10 \mu \mathrm{g} / \mathrm{kg}$, the replacement dose of estradiol in rodents) (27). Suppression of glutathione, glutathione reductase, thioredoxin, and thioredoxin reductase, and their normalization by exogenous $17-\beta$ estradiol, was also observed in the bone marrow of mice (data not shown). Thus, physiological levels of estrogen maintain thiol antioxidants in rodent bone marrow.

Glutathione, glutathione reductase, thioredoxin, and thioredoxin reductase were unchanged in liver or spleen by ovariectomy (data not shown), suggesting a receptormediated action in bone marrow. The equally oxidant but receptor-inactive stereoisomer $17-\alpha$ estradiol did not restore glutathione levels in rodent bone marrow (Figure 1). These considerations suggest that physiological levels of estrogen maintain thiol antioxidants in bone marrow through a receptor-mediated action.

To determine whether the estrogen-induced changes in thiol antioxidants were causally related to bone loss, we tested the effects of agents that increase levels of reduced glutathione (16) on ovariectomy-induced bone loss. We found (Figure 2) that both NAC and ascorbate prevented bone loss in ovariectomized mice. Both osteoclasts in bone, an increase in the proportion of bone surfaces covered by osteoclasts, and an increase in the extent of bone surfaces that showed an eroded appearance. There was also an increase in the number of osteoblasts and an increase in the rate of bone formation. These changes in bone histodynamics are identical to those that are observed after ovariectomy, in which there is an increase in bone resorption that entrains a reactive increase in bone formation insufficient to replace the bone lost.

Although the above experiments suggest that $17-\beta$ estradiol maintains bone quantity through induction of thiol antioxidants, they do not distinguish between central and local mechanisms for this effect on bone, nor, if the action is local, do they identify the target cell in bone. We therefore tested the effects of $17-\beta$ estradiol on the thiol antioxidant system in osteoblastic and osteoclastic cells. We found that neither glutathione reductase nor total glutathione were influenced by incubation in $17-\beta$ estradiol (see Table 1 ). In contrast, $17-\beta$ estradiol strongly stimulated glutathione and thioredoxin reductases in osteoclast-like cells in vitro (Figure 4). This was completely reversed by ICI 182,780 . The $17-\beta$ estradiol also stimulated glutathione levels in osteoclasts (GSH, vehicle: $25.0 \pm 0.9$ $\mathrm{nmol} / \mathrm{mg}$ protein; $17-\beta$ estradiol $\left(10^{-9} \mathrm{M}\right) 29.3 \pm 0.8$ $\mathrm{nmol} / \mathrm{mg}$ protein; GSSG, vehicle: $0.6 \pm 0.2 \mathrm{nmol} / \mathrm{mg}$ protein; $17-\beta$ estradiol $0.5 \pm 0.1 \mathrm{nmol} / \mathrm{mg}$ protein). Moreover, the effect of BSO and NAC on osteoclastic numbers in vitro paralleled their effects in vivo, and we found that the ROS hydrogen peroxide enhanced 


\section{Figure 2}

Ascorbate and NAC prevent bone loss in ovariectomized mice. (a-g) Ascorbate (2 $\mathrm{mmol} / \mathrm{kg} /$ day) prevents ovariectomy-induced bone loss in mice. (a) Representative images of microscope sections of femora from mice subjected to sham ovariectomy (sham) or ovariectomy (ovx) and treated for 2 weeks with ascorbate $(1 \mathrm{nmol} / \mathrm{kg}$ twice daily) or vehicle. (b-g) Histomorphometric analysis shows that ascorbate prevented ovariectomyinduced bone loss (b). Indices of bone resorption (c-e) (osteoclast number per millimeter of bone surface; percentage of bone surface covered by osteoclasts; percentage of bone surface that shows scalloped, eroded appearance) were increased by ovariectomy and suppressed by ascorbate. Ascorbate also normalized ( $\mathbf{f}$ and $\mathbf{g}$ ) the number of osteoblasts per millimeter of bone surface and the percentage of bone surface covered by osteoblasts to sham levels. Total glutathione in bone marrow fell significantly $(P<0.05)$ from $32 \pm 5 \mathrm{nmol} / \mathrm{mg}$ protein in controls to $22 \pm 1.2$ $\mathrm{nmol} / \mathrm{mg}$ protein in ovariectomized mice and was increased by ascorbate to $54 \pm 12.3$ $\mathrm{nmol} / \mathrm{mg}$ protein. (h-m) NAC $(100 \mathrm{mg} / \mathrm{kg} /$ day) prevents ovariectomy-induced bone loss in mice. Indices of bone resorption and bone formation show that while ovariectomy caused a reduction in bone volume $(\mathbf{h})$, this was prevented by NAC. NAC also normalized the number of osteoclasts per millimeter of the bone surface (i), the percentage of bone surface that was covered by osteoclasts (j), and the percentage of bone surface that showed a crenated, eroded surface characteristic of osteoclastic activity (k). NAC also reversed the ovariectomy-induced increase in osteoblast numbers (I) and the percentage of surface that was covered by osteoblasts $(\mathbf{m})$. ${ }^{*} P<0.05$ versus other groups. Data expressed as mean \pm SEM. Oc, osteoclast; ES/BS (\%), percentage of bone surface that shows an eroded surface appearance; Ob, osteoblast.
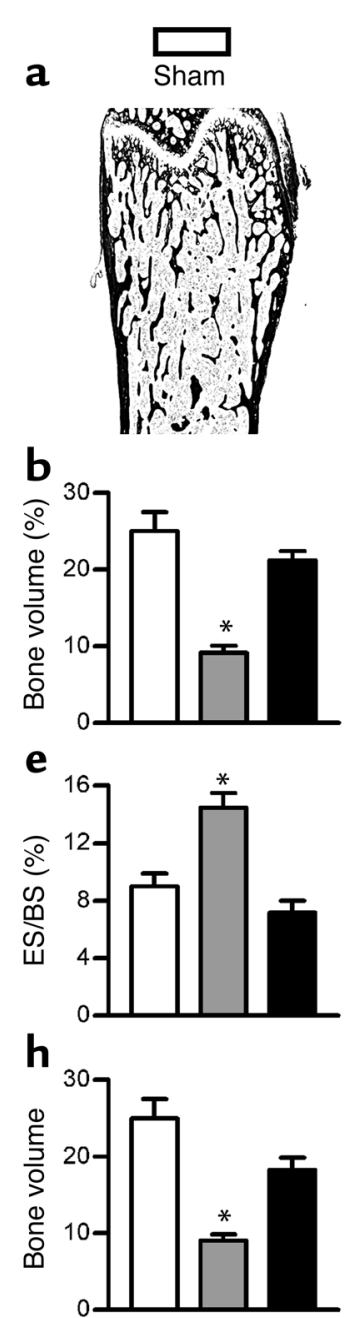

k

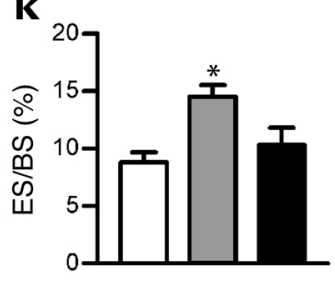

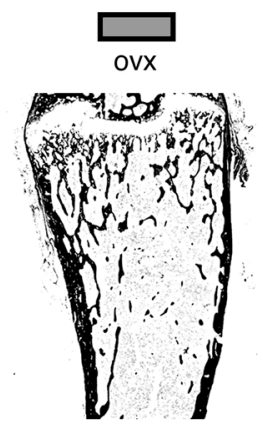

C

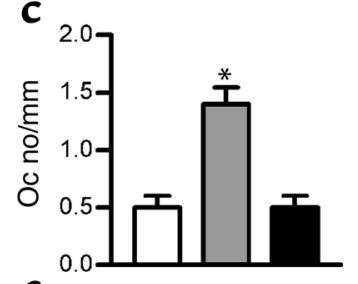

f
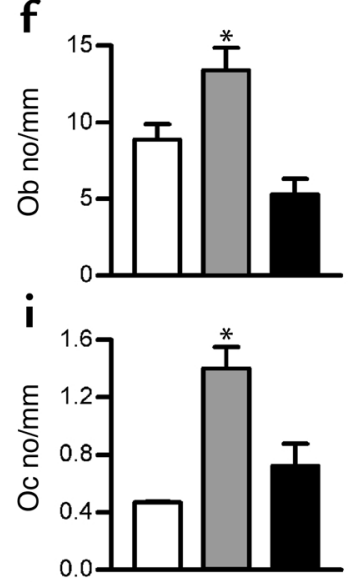

I

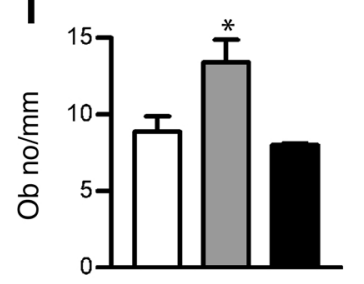

ovx + ascorbate
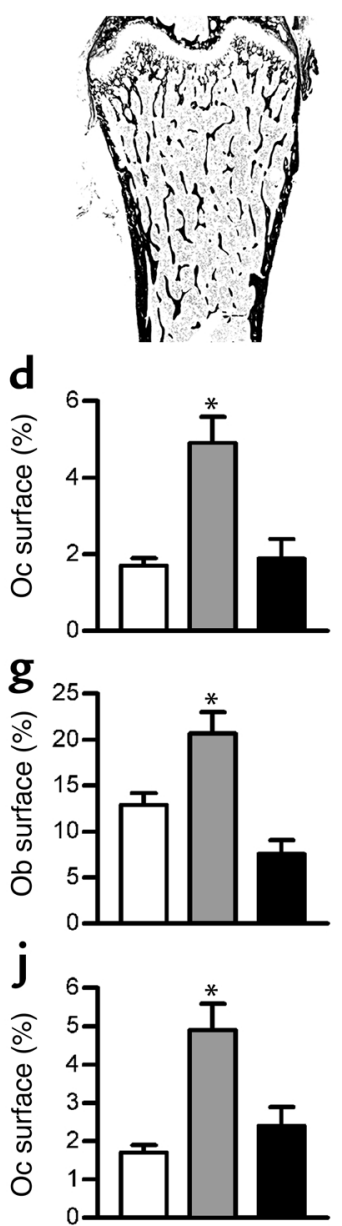

m

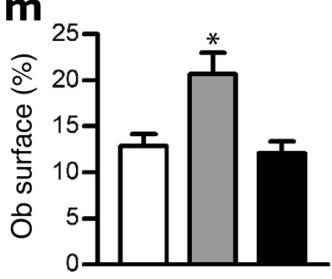

osteoclastic differentiation (Figure 4) in addition to its documented ability to activate osteoclasts (17).

If estrogen does regulate osteoclasts through thiol antioxidants, we would predict that $17-\beta$ estradiol and thiol-modulating agents should influence crucial ROS-sensitive signals in osteoclastic cells. The beststudied signaling target for ROS is NF- $\mathrm{KB}$. Release from its inhibitory complex with IкB is augmented by ROS in many cells. We therefore challenged osteoclastlike cells with BSO or NAC in vitro. We found that BSO augmented, while NAC suppressed, the level of activated NF-kB (Figure 4).

TNF- $\alpha$ is a target cytokine for NF- $\mathrm{KB}$. The cytokine is known to synergize strongly with RANKL for osteoclast formation and has been strongly implicated in the bone loss of estrogen deficiency. Therefore, we tested the effects of estrogen and ROS modulators on TNF- $\alpha$ expression. We found that $17-\beta$ estradiol, like the antioxidant NAC, suppressed expression of mRNA for TNF- $\alpha$ in osteoclasts (Figure 4).

\section{Discussion}

We found that ovariectomy caused a substantial decrease in the level of glutathione and thioredoxin, the major tissue thiol antioxidants, and in the enzymes that regenerate their reduced forms in rodent bone marrow. Both antioxidants and their regenerative enzymes were rapidly normalized by a single dose of $17-\beta$ estradiol $(10 \mu \mathrm{g} / \mathrm{kg}$, the replacement dose in rodents) (27), but not by its non-receptor-binding stereoisomer $17-\alpha$ estradiol. We also found that antioxidants prevented estrogen-deficiency bone loss, while BSO, which depletes thiol antioxidants, induced bone loss. Last, we found evidence for 


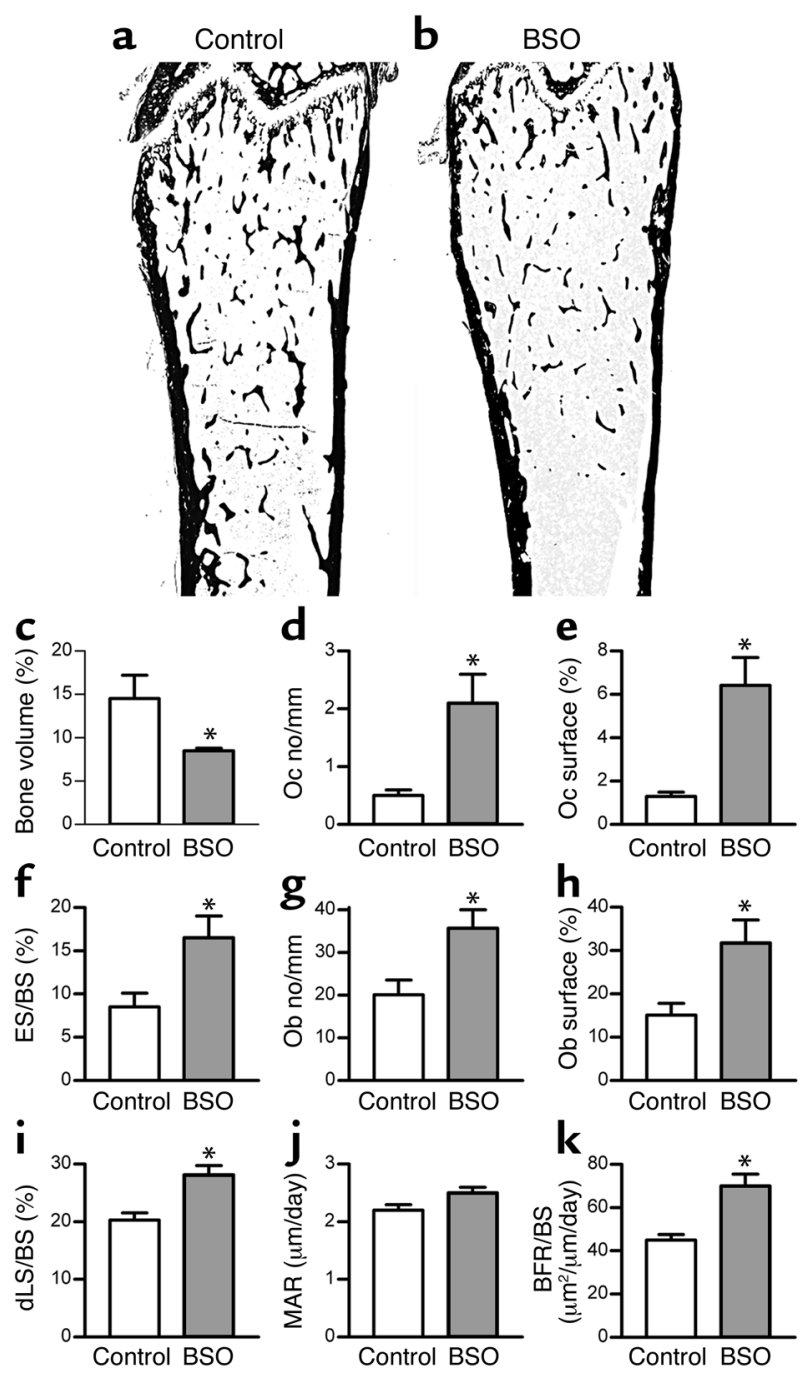

a mechanistic link between ROS and hyper-resorption: estrogen enhanced antioxidant defenses in osteoclasts and, like ROS, modulated expression of the key resorptive cytokine TNF- $\alpha$.

Our results suggest that the effects of $17-\beta$ estradiol on thiol antioxidants occurs via the estrogen receptor: the receptor-inactive stereoisomer $17-\alpha$ estradiol did not normalize thiol antioxidants, and defenses were modulated in bone but not in liver or spleen. We also found that oxidant defenses were lowered by the removal of a physiological source of estrogen and were normalized by very low exogenous doses. These results suggest that physiological levels of estrogen maintain thiol antioxidants in rodent bone.

Thiol antioxidants are major regulators of cell signaling $(13,14)$, so that the decrease caused by estrogen deficiency is likely to influence signaling in bone cells and might thereby be the cause of the associated bone loss. We found that NAC and ascorbate, which raise tissue glutathione concentrations (16, 26), prevented bone loss in ovariectomized mice. Conversely, BSO, like the ovariectomy, lowered thiol antioxidants and caused bone loss. Indeed, BSO

\section{Figure 3}

BSO induces bone loss in mice. ( $\mathbf{a}$ and $\mathbf{b}$ ) Representative sections of femora from a control mouse and a mouse injected with BSO (2 $\mathrm{mmol} / \mathrm{kg}$ ) twice a day for 3 weeks, showing loss of trabecular bone in BSO-treated mouse. (c) BSO caused substantial and significant loss of bone. (d-f) Bone loss was accompanied by an increase in the number of osteoclasts per millimeter of bone surface, the percentage of bone surface covered by osteoclasts, and the percentage of bone surface that showed an eroded surface. ( $\mathbf{g}$ and $\mathbf{h}$ ) BSO also significantly increased the number of osteoblasts covering bone surfaces and the percentage of bone surface covered by osteoblasts. (i-k) Dynamic parameters of bone formation: BSO caused a significant increase in the percentage of bone surface that was actively forming bone matrix, as judged by the percentage of surface that incorporated double calcein labels (i). MAR (mineral apposition rate, measured as the distance of separation of the double labels) was not significantly increased (j). There was an overall increase in the quantity of bone formed per unit of time (bone formation rate per unit of bone surface) (k). ${ }^{*} P<0.05$ versus vehicle-injected control; $n=6$ mice per group. Data expressed as mean \pm SEM. Total glutathione fell significantly $(P<0.01)$ in the bone marrow of BSO-treated mice from $14.5 \pm 1.1$ to $6.8 \pm 0.1 \mathrm{nmol} / \mathrm{mg}$ protein. There was no significant change in body weight in either group of mice during the experimental period. Uterine weights of BSO-treated mice did not differ significantly from those of control mice. Oc, osteoclast; ES/BS (\%), percentage of bone surface that shows an eroded surface appearance; Ob, osteoblast; dLS/BS, proportion of bone surface that shows fluorochrome double-labeling; BFR/BS, bone formation rate expressed as the volume of bone formed per unit of bone surface.

administration provides a phenocopy of estrogendeficiency bone loss: both combine an increase in resorption with a hyperplastic osteoblastic reaction to the hyper-resorption that is inadequate to replace the lost bone. Furthermore, glutathione levels were comparable after ovariectomy and BSO administration. This implies that the degree to which ovariectomy lowered glutathione was sufficient to account for the bone loss of estrogen deficiency. These experiments provide strong evidence that the decrease in thiol antioxidants induced by ovariectomy is causally related to the bone loss that ensues.

Our experiments identified osteoclasts as a target for the augmentation of oxidant-defense enzymes by estradiol. This action will be expected to modulate

\section{Table 1}

$17-\beta$ estradiol does not modulate glutathione reductase or total glutathione levels in osteoblastic cells

$\begin{array}{lccc}\text { Cell type } & \text { Treatment } & \begin{array}{c}\text { Glutathione reductase } \\ (\mathrm{mU} / \mathrm{mg} \text { protein })\end{array} & \begin{array}{c}\text { Total glutathione } \\ (\mathrm{nmol} / \mathrm{\mu g} \text { protein })\end{array} \\ \text { Calvarial cells } & \text { Vehicle } & 25.1 \pm 1.0 & 10.8 \pm 0.5 \\ & \mathrm{E}_{2} & 27.4 \pm 3.6 & 8.5 \pm 0.8 \\ \text { MC-3T3-E1 } & \text { Vehicle } & 16.8 \pm 6.0 & 18.7 \pm 2.1 \\ & \mathrm{E}_{2} & 18.6 \pm 4.1 & 22.0 \pm 1.3 \\ \text { UMR-106 } & \text { Vehicle } & 15.6 \pm 4.6 & 9.7 \pm 1.6 \\ & \mathrm{E}_{2} & 11.8 \pm 1.6 & 11.7 \pm 3.0\end{array}$

Osteoblastic cells were incubated for 18 hours in $17-\beta$ estradiol $\left(10^{-9} \mathrm{M}\right)$ or vehicle before harvesting and assessing levels of glutathione reductase and total glutathione. Data expressed as mean \pm SEM of three cultures per variable. None of the differences are statistically significant. $E_{2}, 17-\beta$ estradiol. 


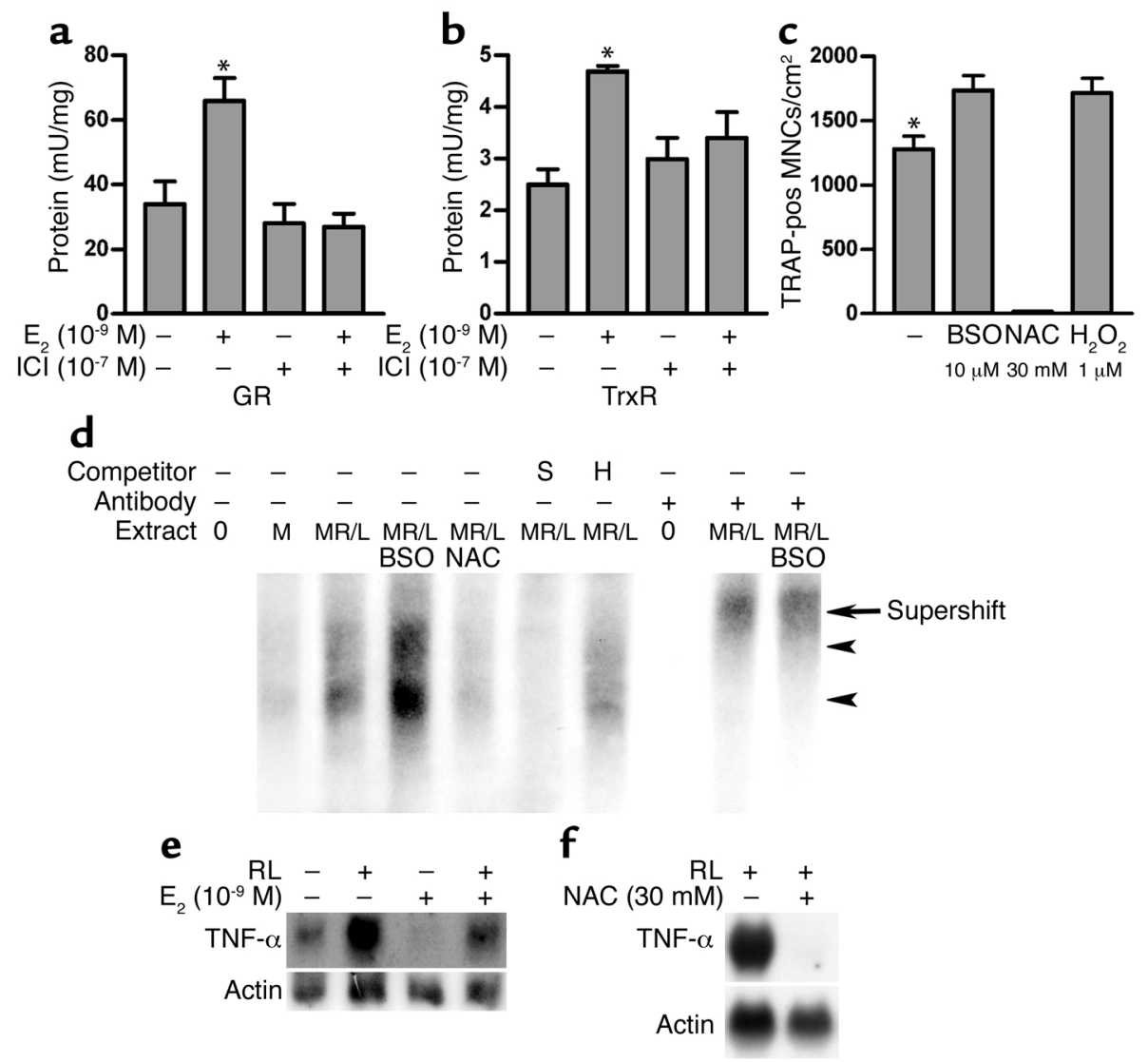

Figure 4

$17-\beta$ estradiol modulates osteoclastic thiol antioxidant system, and osteoclasts are influenced by modulation of thiol antioxidant system. (a and b) 17- $\beta$ estradiol stimulated glutathione reductase (GR) and thioredoxin reductase (TrxR) in osteoclasts. This stimulation was reversed by $\mathrm{ICI} 182,780(\mathrm{ICI})$ (three cultures per variable). Statistically, significant stimulation of GR and TrxR by $17-\beta$ estradiol ( $\left.\mathrm{E}_{2}\right)$ and inhibition of stimulation by ICI 182,780 was observed in each of two further experiments. (c) BSO stimulated TRAP-positive multinucleate cell formation (TRAP-pos MNC), while this was suppressed by NAC. Like BSO, the ROS hydrogen peroxide also stimulated TRAP-positive multinucleate cell formation (10 cultures per variable). (d) EMSA was used to show effect of BSO and NAC on NF-KB activity in in vitro-formed osteoclasts. Activity (arrowheads) was increased by BSO and suppressed by NAC. We confirmed that the bound material contained NF- $\mathrm{KB}$ $\mathrm{p} 50$ by supershifting both bands with p50 Ab (arrow). The binding was further confirmed to be specific by competing binding of NF-KB with 100 -fold excess of unlabeled self probe (S), but not by a mutant species (H). M, M-CSF; RL, RANKL. (e and $\mathbf{f}$ ) 17- $\beta$ estradiol and NAC suppress expression of RNA for TNF- $\alpha$ in in vitro-derived osteoclasts. ${ }^{*} P<0.05$ versus other groups.

ROS-sensitive pathways in osteoclasts. In fact, osteoclasts are a prime candidate for regulation by ROS. Not only do they express NADPH oxidase (20), an enzyme capable of the cytokine-regulated generation of ROS, and generate ROS during bone resorption (18), but their activity is dependent upon several intracellular signals that are sensitive to ROS, including NF- $\mathrm{KB}, \mathrm{JNK}, \mathrm{PI} 3 \mathrm{~K}$, and P38 MAP kinase. Consistent with this, we found that BSO and NAC reciprocally regulated NF- $\mathrm{\kappa B}$ in osteoclasts, as in several other cell types. It may well be that other signaling cascades are similarly influenced in osteoclasts by ROS, thereby providing further mechanisms through which modulation of oxidant defenses by estrogen might directly modulate osteoclastic activity.

In addition to the direct effects of ROS on these signaling cascades in osteoclasts, ROS might also indirectly stimulate osteoclasts by augmenting expression of resorptive cytokines such as TNF- $\alpha$, IL-1, and
IL-6 that have been strongly implicated in estrogendeficiency bone loss (31-33). ROS are potent inducers of these cytokines in many cells through activation of NF-KB (see ref. 34). We found that ROS similarly activate NF- $\mathrm{\kappa B}$ in osteoclasts and that estrogen, which raises antioxidant defenses, and the antioxidant NAC strongly suppress TNF- $\alpha$ expression in osteoclastic cells in vitro. Recently, it was shown that TNF- $\alpha$ synergizes strongly with RANKL for osteoclast formation and activation $(28,35)$ and that mice lacking TNF- $\alpha$ signaling do not lose bone after ovariectomy (33). Therefore, ROS might induce bone hyper-resorption in estrogen deficiency through the autocrine-paracrine effects of ROS-augmented TNF- $\alpha$ expression in osteoclasts.

Bone hyper-resorption can thus be attributed to the direct and indirect effects of estrogen deficiency on thiol antioxidants in osteoclasts. This does not preclude, however, similar responses to estrogen in 
endothelial, macrophagic, lymphoid, or other cells in bone marrow. Such cells might also be affected indirectly through the ROS that osteoclasts have been shown to generate: some ROS, particularly hydrogen peroxide, are sufficiently stable and membrane permeant to transmit ROS signals to nearby cells. Glutathione reductase is rate limiting for elimination of hydrogen peroxide (36), and thioredoxin itself degrades hydrogen peroxide (37-39). The depressed bone marrow thiol antioxidant system we have observed might thereby enhance expression of resorptive cytokines in other bone marrow cells. Moreover, ROS and TNF- $\alpha$ not only stimulate resorption but suppress osteoblastic differentiation (40-43) as well. Thus, if the lowering of oxidant defenses in bone marrow by estrogen deficiency does cause an increase in hydrogen peroxide and/or TNF- $\alpha$ in the bone marrow space, these might contribute to the defect in osteoblastic function that is observed in estrogen-deficient states. We observed a similar osteoblastic deficit after BSO administration. ROS may perturb osteoblastic function in additional ways; for example, ROS inactivate nitric oxide, deficiency of which impairs bone formation in vivo (44).

The notion that oxidative status is important in osteoporosis is consistent with the strong epidemiological association between osteoporosis and atherosclerosis, a disease in which oxidant stress plays a major role. Moreover, it was recently observed that there is an association in women between oxidant stress (measured by a biomarker of oxidative stress, 8 -iso- $\mathrm{PGF}_{2}-\alpha$ ) (45), antioxidant levels (46), and bone mineral density. Clinical studies that have examined the relationship between dietary and/or supplemental ascorbate and bone mass in humans also suggest an association with increased bone mass (47).

These results have important implications for bone biology and the treatment of osteoporosis. If the model is correct, it predicts not only the bone loss of estrogen deficiency, but also predicts that bone loss seen in other situations in which ROS have been implicated, such as in aging and inflammation, might be caused by a prolonged augmentation of ROS signaling in bone cells. Thus, ROS generated by inflammatory tissue in or adjacent to bone might cause the loss of bone that is a feature of diseases such as rheumatoid arthritis or periodontitis. Furthermore, osteoporosis has recently been noted in two mouse models of premature aging associated with oxidative damage $(48,49)$ in which osteopenia is presumed to be the consequence of oxidative damage. It may be that estrogen deprivation, by lowering tissue thiol antioxidant levels, leads to oxidative damage and that this, rather than signal modulation, causes bone loss. Alternatively, signal modulation by the increased oxidant stress in these models of premature aging might account for the bone loss. Whichever mechanism underlies the bone loss, our results predict that osteoporosis should be prevented by therapies that increase oxidant defenses in bone.

\section{Acknowledgments}

This work was supported by the Wellcome Trust and the Arthritis and Rheumatism Council.

1. Riggs, B.L., Khosla, S., and Melton, L.J., III. 2002. Sex steroids and the construction and conservation of the adult skeleton. Endocr. Rev. 23:279-302.

2. Shevde, N.K., Bendixen, A.C., Dienger, K.M., and Pike, J.W. 2000. Estrogens suppress RANK ligand-induced osteoclast differentiation via a stromal cell independent mechanism involving c-Jun repression. Proc. Natl. Acad. Sci. U. S. A. 97:7829-7834.

3. Parikka, V., et al. 2001. Estrogen reduces the depth of resorption pits by disturbing the organic bone matrix degradation activity of mature osteoclasts. Endocrinology. 142:5371-5378.

4. Srivastava, S., et al. 2001. Estrogen decreases osteoclast formation by down-regulating receptor activator of NF-kappa B ligand (RANKL)induced JNK activation. J. Biol. Chem. 276:8836-8840.

5. Eghbali-Fatourechi, G., et al. 2003. Role of RANK ligand in mediating increased bone resorption in early postmenopausal women. J. Clin. Invest. 111:1221-1230. doi:10.1172/JCI200317215.

6. Hofbauer, L.C., et al. 1999. Estrogen stimulates gene expression and protein production of osteoprotegerin in human osteoblastic cells. Endocrinology. 140:4367-4370.

7. Manologas, S.C., Kousteni, S., and Jilka, R.L. 2002. Sex steroids and bone. Recent Prog. Horm. Res. 57:385-409.

8. Pfeilschifter, J., Koditz, R., Pfohl, M., and Schatz, H. 2002. Changes in proinflammatory cytokine activity after menopause. Endocr. Rev. 23:90-119.

9. Sack, M.N., Rader, D.J., and Cannon, R.O. 1994. Oestrogen and inhibition of oxidation of low-density lipoproteins in postmenopausal women. Lancet. 343:269-270.

10. Sudoh, N., et al. 2001. Estrogen prevents oxidative stress-induced endothelial cell apoptosis in rats. Circulation. 103:724-729.

11. Arnal, J.F., et al. 1996. Ethinylestradiol does not enhance the expression of nitric oxide synthase in bovine endothelial cells but increases the release of bioactive nitric oxide by inhibiting superoxide anion production. Proc. Natl. Acad. Sci. U. S. A. 93:4108-4113.

12. Sawada, H., et al. 2000. Mechanisms of antiapoptotic effects of estrogens in nigral dopaminergic neurons. FASEB J. 14:1202-1214.

13. Dröge, W. 2002. Free radicals in the physiological control of cell function. Physiol. Rev. 82:47-95.

14. Haddad, J.J. 2002. Antioxidant and prooxidant mechanisms in the regulation of redox(y)-sensitive transcription factors. Cell Signal. 14:879-897.

15. Paolicchi, A., Dominici, S., Pieri, L., Maellaro, E., and Pompella, A. 2002. Glutathione catabolism as a signaling mechanism. Biochem. Pharmacol. 64:1027-1035.

16. Meister, A. 1994. Glutathione-ascorbic acid antioxidant system in animals. J. Biol. Chem. 269:9397-9400.

17. Bax, B.E., et al. 1992. Stimulation of osteoclastic bone resorption by hydrogen peroxide. Biochem. Biophys. Res. Comm. 183:1153-1158.

18. Garrett, I.R., et al. 1990. Oxygen-derived free radicals stimulate osteoclastic bone resorption in rodent bone in vitro and in vivo. J. Clin. Invest. 85:632-639.

19. Finkel, T., and Holbrook, N.J. 2000. Oxidants, oxidative stress and the biology of ageing. Nature. 408:239-247.

20. Steinbeck, M.J., Appel, W.H., Jr., Verhoeven, A.J., and Karnovsky, M.J. 1994. NADPH-oxidase expression and in situ production of superoxide by osteoclasts actively resorbing bone. J. Cell Biol. 126:765-772.

21. Dickinson, D.A., and Forman, H.J. 2002. Cellular glutathione and thiols metabolism. Biochem. Pharmacol. 64:1019-1026.

22. Danon, A. 2002. Redox reactions of regulatory proteins: do kinetics promote specificity? Trends Biochem. Sci. 27:197-203.

23. Tietze, F. 1969. Enzymic method for quantitative determination of nanogram amounts of total and oxidized glutathione: applications to mammalian blood and other tissues. Anal. Biochem. 27:502-522.

24. Baker, M.A., Cerniglia, G.J., and Zaman, A. 1990. Microtiter plate assay for the measurement of glutathione and glutathione disulfide in large numbers of biological samples. Anal. Biochem. 190:360-365.

25. Holmgren, A., and Björnstedt, M. 1995. Thioredoxin and thioredoxin reductase. Methods. Enzymol. 252:199-208.

26. Jain, A., et al. 1992. Ascorbic acid prevents oxidative stress in glutathionedeficient mice: effects on lung type 2 cell lamellar bodies, lung surfactant, and skeletal muscle. Proc. Natl. Acad. Sci. U. S. A. 89:5093-5097.

27. Chow, J., Tobias, J.H., Colston, K.W., and Chambers, T.J. 1992. Estrogen maintains trabecular bone volume in rats not only by suppression of bone resorption but also by stimulation of bone formation. J. Clin. Invest. 89:74-78.

28. Fuller, K., Murphy, C., Kirstein, B., Fox, S.W., and Chambers, T.J. 2002. TNF $\alpha$ potently activates osteoclasts, through a direct action independent of and strongly synergistic with RANKL. Endocrinology. 143:1108-1118. 
29. Partington, G.A., and Patient, R.K. 1999. The DNA-binding affinity of GATA- 1 is altered by changes in phosphorylation during erythroleukemia cell differentiation. Nucleic Acids Res. 27:1168-1175.

30. Lean, J.M., et al. 2000. Osteoclast lineage commitment of bone marrow precursors through expression of membrane-bound TRANCE. Bone. 27:29-40.

31. Jilka, R.L., et al. 1992. Increased osteoclast development after estrogen loss: mediation by interleukin-6. Science. 257:88-91.

32. Kitazawa, R., Kimble, R.B., Vannice, J.L., Kung, V.T., and Pacifici, R. 1994. Interleukin-1 receptor antagonist and tumor necrosis factor binding protein decrease osteoclast formation and bone resorption in ovariectomized mice. J. Clin. Invest. 94:2397-2406.

33. Roggia, C., et al. 2001. Up-regulation of TNF-producing T cells in the bone marrow: a key mechanism by which estrogen deficiency induces bone loss in vivo. Proc. Natl. Acad. Sci. U. S. A. 98:13960-13965.

34. Garg, A.K., and Aggarwal, B.B. 2002. Reactive oxygen intermediates in TNF signaling. Mol. Immunol. 39:509-517.

35. Lam, J., et al. 2000. TNF- $\alpha$ induces osteoclastogenesis by direct stimulation of macrophages exposed to permissive levels of RANK ligand. J. Clin. Invest. 106:1481-1488.

36. Sasaki, K., Bannai, S., and Makino, N. 1998. Kinetics of hydrogen peroxide elimination by human umbilical vein endothelial cells in culture. Biochim. Biophys. Acta. 1380:275-288.

37. Mitsui, A., Hirakawa, T., and Yodoi, J. 1992. Reactive oxygen-reducing and protein-refolding activities of adult $\mathrm{T}$ cell leukemia-derived factor/human thioredoxin. Biochem. Biophys. Res. Comm. 186:1220-1226.

38. Nordberg, J., and Arnér, E.S.J. 2001. Reactive oxygen species, antioxidants, and the mammalian thioredoxin system. Free Radic. Biol. Med. 31:1287-1312.
39. Spector, A., et al. 1988. The effect of $\mathrm{H}_{2} \mathrm{O}_{2}$ upon thioredoxin-enriched lens epithelial cells. J. Biol. Chem. 263:4984-4990.

40. Mody, N., Parhami, F., Sarafian, T.A., and Demer, L.L. 2001. Oxidative stress modulates osteoblastic differentiation of vascular and bone cells. Free Radic. Biol. Med. 31:509-519.

41. Gilbert, L., et al. 2002. Expression of the osteoblast differentiation factor RUNX2 (Cbfa1/AML3/Pebp2alpha A) is inhibited by tumor necrosis factor-alpha. J. Biol. Chem. 277:2695-2701.

42. Panagakos, F.S., Fernandez, C., and Kumar, S. 1996. Ultrastructural analysis of mineralized matrix from human osteoblastic cells: effect of tumor necrosis factor-alpha. Mol. Cell. Biochem. 158:81-89.

43. Samoto, H., et al. 2002. TNF-alpha suppresses bone sialoprotin (BSP) expression in ROS17/2.8 cells. J. Cell Biochem. 87:313-323.

44. Armour, K.E., et al. 2001. Defective bone formation and anabolic response to exogenous estrogen in mice with targeted disruption of endothelial nitric oxide synthase. Endocrinology. 142:760-766.

45. Basu, S., Michaelsson, K., Olofsson, H., Johansson, S., and Melhus, H. 2001. Association between oxidative stress and bone mineral density. Biochem. Biophys. Res. Comm. 288:275-279.

46. Maggio, D., et al. 2003. Marked decrease in plasma antioxidants in aged osteoporotic women: results of a cross-sectional study. J. Clin. Endocrinol. Metab. 88:1523-1527.

47. Morton, D.J., Barrett-Connor, E.L., and Schneider, D.L. 2001. Vitamin C supplement use and bone mineral density in postmenopausal women. J. Bone Miner. Res. 16:135-140.

48. Tyner, S.D., et al. 2002. p53 mutant mice that display early aging-associated phenotypes. Nature. 415:45-53.

49. de Boer, J., et al. 2002. Premature aging in mice deficient in DNA repair and transcription. Science. 296:1276-1279. 